\title{
Susceptibility Artifacts in the Anterior Cranial Fossa Mimicking Hemorrhage in Patients with Anosmia
}

$\mathbf{T}$ here has been a steady stream of requests for MR imaging studies of the brain for investigating anosmia in patients with coronavirus disease 2019 (COVID-19) at our institution in Miami, which was briefly the American center of the pandemic. We read with great interest the report of Aragão et $\mathrm{al}^{1}$ in the September 2020 issue of AJNR, titled "Anosmia in COVID-19 Associated with Injury to the Olfactory Bulbs Evident on MRI," and we have occasionally encountered similar findings in patients with anosmia. In interpreting these examinations, we have assumed (given the limited history available to us) that these patients are either known or presumed to have been infected with COVID-19. As the novelty of these findings has lessened, members of our group have begun to realize similar findings are present in many patients who were referred for unrelated indications. Furthermore, we have ascribed the striking symmetry of the high signal to the presence of methemoglobin. The lack of morphologic (ie, swelling) changes in the areas of high signal and the lack of involvement of the olfactory bulb have led us to question the validity of these findings.

The letter by Dr Mamourian ${ }^{2}$ published in the February 2021 issue of $A J N R$ closely echoes the suspicion of members of our group that the observed areas of high signal were artifactual, likely secondary to magnetic susceptibility artifacts from the airbone interfaces present in the anterior cranial fossa.

In March 2020, a patient underwent 3T MR imaging of her brain at our public hospital for investigation of anosmia. Areas of high T1 signal were present in the anterior cranial fossa bilaterally, localizing symmetrically to the olfactory sulcus and medial orbital gyrus. No abnormality was observed in the olfactory bulb itself (Fig 1). Upon review of the patient's medical records, it was discovered the patient's anosmia greatly predated (by many years) the COVID-19 pandemic and that the patient had antibod- ies negative for COVID-19. This information heightened our suspicion that the T1 shortening observed in this patient and others with similar findings represented magnetic susceptibility artifacts. It was hypothesized that these findings would be absent or less prominent on a repeat examination with a lower strength magnet, as this is a characteristic of magnetic susceptibility artifacts. After a discussion with the referring physician, the patient returned a week later for a repeat examination on a $1.5 \mathrm{~T}$ scanner, and the previously observed findings were absent. This strongly indicates an artifactual origin of these findings (Fig 2).

While COVID-19 has surely become the most prominent and is an increasingly important cause of anosmia in the American population, other causes of anosmia exist. Learning of purported MR imaging findings in the brain correlating with their symptoms might cause patients distress or a worsening of symptoms. It is incumbent upon neuroradiologists to exercise caution in the description of novel findings in novel diseases.

\section{REFERENCES}

1. Aragão MD, Leal MC, Cartaxo FO, et al. Anosmia in COVID-19 associated with injury to the olfactory bulbs evident on MRI. AJNR Am J Neuroradiol 2020;41:1703-06 CrossRef Medline

2. Mamourian A. Seeing what we expect to see in COVID-19. AJNR Am J Neuroradiol 2021;42:E1 CrossRef Medline 


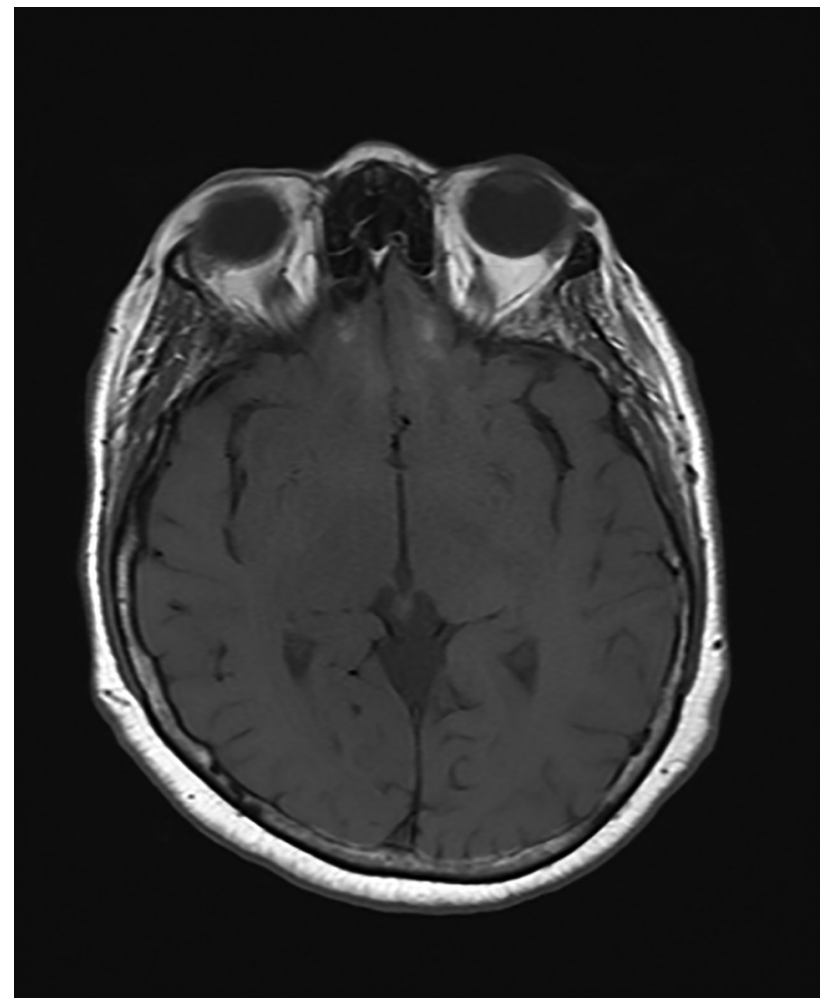

FIG 1. T1-weighted spin-echo sequence obtained on a $3 T$ magnet demonstrating shortening in the olfactory sulcus and medial orbital gyrus.

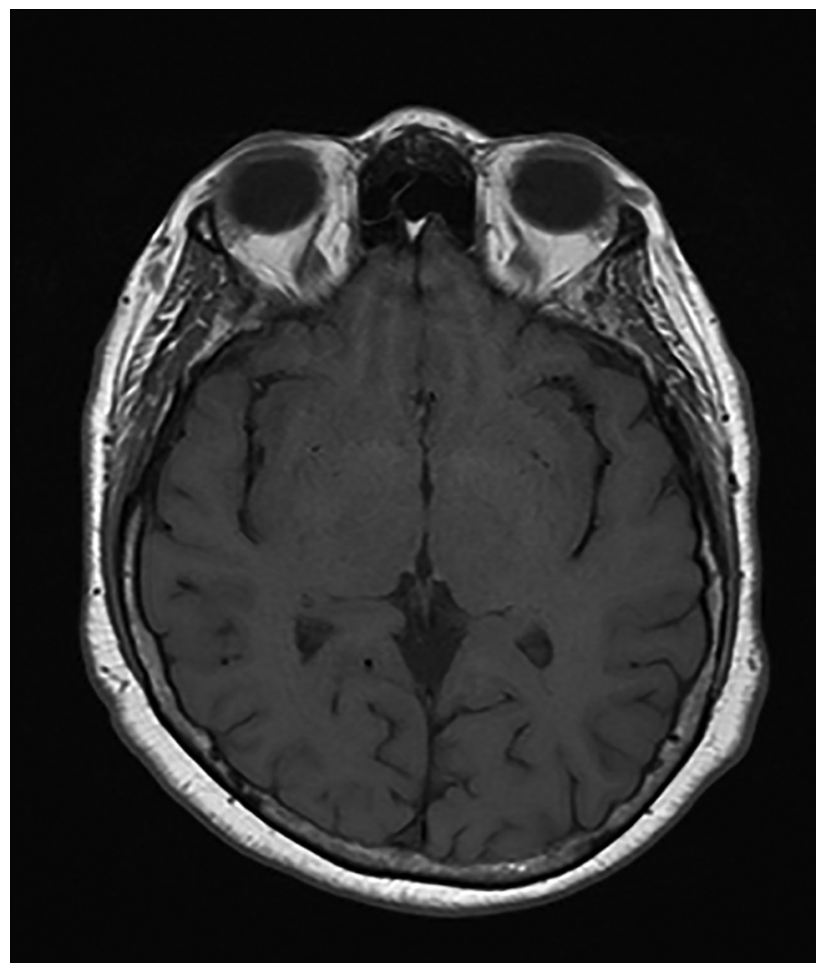

FIG 2. T7-weighted spin-echo sequence obtained on a $1.5 \mathrm{~T}$ magnet with an absence of the previously observed signal. 